\title{
On existence and stability results to a class of boundary value problems under Mittag-Leffler power law
}

\author{
Gauhar Ali ${ }^{1}$, Kamal Shah' ${ }^{1}$, Thabet Abdeljawad ${ }^{2,3,4 *}$, Hasib Khan ${ }^{5 *}$, Ghaus Ur Rahman ${ }^{6}$ and Aziz Khan ${ }^{2}$
}

\section{"Correspondence:}

tabdeljawad@psu.edu.sa; hasibkhan13@yahoo.com

${ }^{2}$ Department of Mathematics and

General Sciences, Prince Sultan

University, Riyadh, Saudi Arabia

${ }^{5}$ Department of Mathematics, Shaheed Benazir Bhutto University, Sheringal, $18000 \operatorname{Dir}(U)$, Pakistan Full list of author information is available at the end of the article

\section{Springer}

\begin{abstract}
Some essential conditions for existence theory and stability analysis to a class of boundary value problems of fractional delay differential equations involving Atangana-Baleanu-Caputo derivative are established. The deserted results are derived by using the Banach contraction and Krasnoselskii's fixed point theorems. Moreover, different kinds of stability theory including Hyers-Ulam, generalized Hyers-Ulam, Hyers-Ulam-Rassias and generalized Hyers-Ulam-Rassias stability are also developed for the problem under consideration. Appropriate examples are given for illustrative purposes.
\end{abstract}

MSC: Primary 26A33; secondary 34A08; 35A08

Keywords: Boundary conditions; $A B C$ fractional derivative; Krasnoselskii fixed point theorem; Stability theory

\section{Introduction}

Fractional-order differential equations (FDEs) have large numbers of application in modeling various real-world processes and phenomena. Due to this researchers have taken a keen interest in the development of the concerned area of research. A valuable contribution in the development of the theory was made by different researchers; see [1-3] and in the references therein. The real-world problems involving the memory effects is one of the biggest challenges for the researchers. Therefore to overcome this deficiency, some new techniques and tools were developed by different researchers to furnish the theory further. The phenomena related to dynamics, thermodynamics, control theory, biophysics, biomedical, computer networking, electrostatics, image and signal processing and economics are commonly modeled via the aforementioned equations [2, 3]. Due to the reliability, a great degree of freedom and global nature of FDEs as compared to traditional differential equations (DEs), researchers paid more attention to the concerned area. In this connection, we refer to [4-9]. The researchers studied different aspects of FDEs. Of course an important aspect of FDEs is the existence of a solution, its uniqueness and stability analysis. They used fixed point theory and different techniques of analysis to investigate the

(c) The Author(s) 2020. This article is licensed under a Creative Commons Attribution 4.0 International License, which permits use sharing, adaptation, distribution and reproduction in any medium or format, as long as you give appropriate credit to the original author(s) and the source, provide a link to the Creative Commons licence, and indicate if changes were made. The images or other third party material in this article are included in the article's Creative Commons licence, unless indicated otherwise in a credit line to the material. If material is not included in the article's Creative Commons licence and your intended use is not permitted by statutory regulation or exceeds the permitted use, you will need to obtain permission directly from the copyright holder. To view a copy of this licence, visit http://creativecommons.org/licenses/by/4.0/. 
stability and existence of solutions for FDEs. In this connection, the researchers published a variety of books and articles [10].

Another important type of DEs is known as delay DEs (DDEs). There are various kinds of DDEs: continuous delay, discrete type delay and proportional type delay. Each delay has its own characteristics in the modeling of real-world problems. The proportional type DDEs constitute an important class and have a large number of applications in dynamical systems and their uses [11]. It frequently occurred in technological control or natural problems. In such a system, the monitoring and adjustment to the system are observed by the controller. As a result, as regards the arising time delay in between the observation and action control, these adjustments of concern cannot be made immediately. Perfect real-life examples that reflect time delay are one of the important tools to determine the dynamics and an essential part of the system is natural networking. Specifically, a time delay occurs in the communication between neurons. It has been observed that the use of time delay in the model of such a system directs to a convoluted dynamics and even disorder [12]. Furthermore, fractional-order DDEs have a variety of applications in diverse fields, such as hydraulic network systems, automatic control systems, transmission lines, economy, and biology (see [13]). The concerned equations have gotten considerable attention of researchers because these described accurately almost all the electro-dynamic and other real-world situations.

There are different types of differential operators, such as the Riemann-Liouville (RL), Caputo, Hadamard, Caputo-Fabrizo (CF), and Atangana-Baleanu-Caputo (ABC) types. The benefits of these various derivatives, which give the freedom of selection to the researchers to choose the best one of them, is that they will accurately describe the situation. The derivatives in the sense of Riemann-Liouville and Caputo are vastly used and have been well explored by different researchers; see [14, 15]. Probably, the classical fractional derivatives involving a singular kernel cannot determine the non-local dynamics always. Therefore researchers introduced some new class of fractional differential operators known as nonsingular derivatives. In 2016, a nonsingular derivative involving an exponential function was introduced by Caputo and Fabrizo. In subsequent years the concerned derivative were generalized by Atangana-Baleanu-Caputo and was named the $A B C$ fractional derivative. The concerned operator has recently been construed non-locally, without singular kernel and reliable differential operator, as applied in modeling of various real-world phenomena [16] regularly. The complex situations due to singular kernel have been replaced by involving exponential and power decay law; for details see $[17,18]$. The problems under $A B C$ derivative have been studied for iterative solutions mostly by using some integral transform, but very rarely have been investigated as regards qualitative aspects.

On the other hand, sometimes it was too complex to obtain an exact solution of a nonlinear FDEs, in such a situation stability analysis plays a vital role in the investigation. There are varieties of stabilities presented in the literature in the past, such as Lyapunov stability, exponential stability, asymptotic stability, and Mittag-Leffler stability [19-21]. Probably, the most reliable stabilities are those known as Hyers-Ulam (HU) stability. The concerned stability was modified to generalized HU stability (see [22-25]). In 1970, the aforementioned stability was further generalized by Rassias [26]. The concerned areas of existence and stability analysis are well furnished for FDEs involving Caputo and Riemann-Liouville operators [27-30]. But in the case of $A B C$ derivative, the very high relay version was inves- 
tigated. For a pantograph type problem it has not been properly investigated yet. Inspired by the aforementioned literature, we investigate the following fractional DDEs under inhomogeneous boundary conditions:

$$
\left\{\begin{array}{l}
{ }_{0}^{A B C} \mathrm{D}_{t}^{\alpha} \omega(t)=f(t, \omega(t), \omega(v t)), \quad 0<v<1,1<\alpha \leq 2, \\
\omega(0)=\omega_{0}, \quad \omega(T)=\omega_{1},
\end{array}\right.
$$

where ${ }_{0}^{A B C} \mathrm{D}_{t}^{\alpha}$ represents the $A B C$ derivative, $f: J \times \mathrm{R} \times \mathrm{R} \rightarrow \mathrm{R}$ is a continuous function and $J=[0, T]$. Ordinary classes of FDEs are well studied and explored as regards the existence and stability analysis via different methods. But pantograph type fractional DDEs are relay versions to be investigated from this point of view. The aforementioned fractional DDEs is to be investigated with the help of $A B C$ fractional-order derivatives. Therefore, we develop conditions for the existence and different types of stabilities with the help of results of nonlinear analysis and fixed point theory. Further we investigate the boundary value problem (BVP) which has many applications in mathematical modeling of numerous processes and phenomena in engineering, physics and dynamics systems. To illustrate the results we give some examples.

\section{Background materials}

This section consist of some basic definitions and lemmas, which are required in this article. Let $X=C[J, \mathrm{R}]$ be a Banach space with norm $\|\omega\|=\max _{t \in J}|\omega(t)|$.

Definition $1([16,18])$ Let $\omega \in H^{1}(a, b), a<b$ and $\alpha \in[0,1]$. The $A B C$ fractional derivative of $\omega$ of order $\alpha$ is defined as

$$
{ }_{a}^{A B C} \mathrm{D}_{t}^{\alpha} \omega(t)=\frac{\mathbb{M}(\alpha)}{1-\alpha} \int_{a}^{t} \omega^{\prime}(\zeta) \mathrm{E}_{\alpha}\left(\frac{-\alpha(t-\zeta)^{\alpha}}{1-\alpha}\right) d \zeta
$$

We use in our paper this definition. And in the Riemann-Liouville sense we define

$$
{ }_{a}^{A B R} \mathrm{D}_{t}^{\alpha} \omega(t)=\frac{\mathbb{M}(\alpha)}{1-\alpha} \frac{d}{d t} \int_{a}^{t} \omega(\zeta) \mathrm{E}_{\alpha}\left(\frac{-\alpha(t-\zeta)^{\alpha}}{1-\alpha}\right) d \zeta
$$

Here $\mathbb{M}(\alpha)>0$ is a normalization function with the property $\mathbb{M}(0)=\mathbb{M}(1)=1$ and $E_{\alpha}$ is the Mittag-Liffler function.

Definition $2([16,18])$ The Atangana-Baleanu fractional integral of $\omega$ of order $\alpha$ is given by

$$
{ }_{a}^{A B} \mathrm{I}_{t}^{\alpha} \omega(t)=\frac{1-\alpha}{\mathbb{M}(\alpha)} \omega(t)+\frac{\alpha}{\mathbb{M}(\alpha) \Gamma(\alpha)} \int_{a}^{t} \omega(\zeta)(t-\zeta)^{\alpha-1} d \zeta
$$

Lemma 1 ([31]) The AB fractional integral and derivative of order $\alpha \in(0,1)$ of the function $\omega$, satisfy

$$
{ }_{a}^{A B} \mathrm{I}_{t a}^{\alpha A B C} \mathrm{D}_{t}^{\alpha} \omega(t)=\omega(t)-\omega(a) .
$$


Definition 3 ([31]) Let $\omega$ be a function such that $\omega^{n} \in H^{1}(a, b)$ and $n<\alpha \leq n+1$. Then the $A B C$ derivative is

$$
{ }_{a}^{A B C} \mathrm{D}_{t}^{\alpha} \omega(t)={ }_{a}^{A B C} \mathrm{D}_{t}^{\beta} \omega^{(n)}(t) .
$$

And the associated integral is given as

$$
{ }_{a}^{A B} I_{t}^{\alpha} \omega(t)={ }_{a} I_{t a}^{n A B} I_{t}^{\beta} \omega(t) .
$$

Here $\beta=\alpha-n$.

Lemma 2 ([31]) The AB fractional integral and derivative of order $\alpha \in(n, n+1)$ of the function $\omega$, satisfy

$$
{ }_{a}^{A B} \mathrm{I}_{t a}^{\alpha A B C} \mathrm{D}_{t}^{\alpha} \omega(t)=\omega(t)-\sum_{k=0}^{n} \frac{\omega^{(k)}(a)}{k !}(t-a)^{k}
$$

Theorem 1 (Krasnoselskii's fixed-point theorem [32]) If $V \subset X$ be a convex and closed non-empty subset, there exist two operators $F, G$ such that

- $F v_{1}+G v_{2} \in V$ for all $v_{1}, v_{2} \in V$,

- $F$ is a condensing operator,

- $G$ is continuous and compact,

then there exists at least one solution $v \in E$ such that

$$
F(v)+G(v)=v .
$$

\section{Main results}

In this section, we examine the existence and uniqueness of solutions of our proposed problem (1).

Lemma 3 Let $y \in J$, then the solution of the problem

$$
\left\{\begin{array}{l}
{ }_{a}^{A B C} \mathrm{D}_{t}^{\alpha} \omega(t)=y(t), \quad 1<\alpha \leq 2, t \in[0, T], \\
\omega(0)=\omega_{0}, \quad \omega(T)=\omega_{1},
\end{array}\right.
$$

is given by

$$
\begin{aligned}
\omega(t)= & \frac{t w_{1}+w_{0}(T-t)}{T}-\frac{t(2-\alpha)}{T \mathbb{M}(\alpha-1)} \int_{0}^{T} y(\zeta) d \zeta \\
& -\frac{t(\alpha-1)}{T \mathbb{M}(\alpha-1) \Gamma(\alpha)} \int_{0}^{T} y(\zeta)(T-\zeta)^{\alpha-1} d \zeta \\
& +\frac{2-\alpha}{\mathbb{M}(\alpha-1)} \int_{0}^{t} y(\zeta) d \zeta+\frac{\alpha-1}{\mathbb{M}(\alpha-1) \Gamma(\alpha)} \int_{0}^{t} y(\zeta)(t-\zeta)^{\alpha-1} d \zeta
\end{aligned}
$$

Proof By applying the integral ${ }_{0}^{A B} I_{t}^{\alpha}$ to (5), we have

$$
\omega(t)=c_{0}+c_{1}+\frac{2-\alpha}{\mathbb{M}(\alpha-1)} \int_{0}^{t} y(\zeta) d \zeta+\frac{\alpha-1}{\mathbb{M}(\alpha-1) \Gamma(\alpha)} \int_{0}^{t} y(\zeta)(t-\zeta)^{\alpha-1} d \zeta .
$$


Using $w(0)=w_{0}$ and $w(T)=w_{1}$ in (6) implies $c_{0}=w_{0}$ and

$$
c_{1}=\frac{w_{1}-w_{0}}{T}-\frac{2-\alpha}{T \mathbb{M}(\alpha-1)} \int_{0}^{T} y(\zeta) d \zeta-\frac{\alpha-1}{T \mathbb{M}(\alpha-1) \Gamma(\alpha)} \int_{0}^{T} y(\zeta)(T-\zeta)^{\alpha-1} d \zeta
$$

By putting the value of $c_{0}$ and $c_{1}$ in (6), we get

$$
\begin{aligned}
\omega(t)= & \frac{t w_{1}+w_{0}(T-t)}{T}-\frac{t(2-\alpha)}{T \mathbb{M}(\alpha-1)} \int_{0}^{T} y(\zeta) d \zeta \\
& -\frac{t(\alpha-1)}{T \mathbb{M}(\alpha-1) \Gamma(\alpha)} \int_{0}^{T} y(\zeta)(T-\zeta)^{\alpha-1} d \zeta \\
& +\frac{2-\alpha}{\mathbb{M}(\alpha-1)} \int_{0}^{t} y(\zeta) d \zeta+\frac{\alpha-1}{\mathbb{M}(\alpha-1) \Gamma(\alpha)} \int_{0}^{t} y(\zeta)(t-\zeta)^{\alpha-1} d \zeta
\end{aligned}
$$

Corollary 1 In view of Lemma 3, our considered problem (1) is equal to the following integral equation:

$$
\begin{aligned}
\omega(t)= & \frac{t w_{1}+w_{0}(T-t)}{T}-\frac{t(2-\alpha)}{T \mathbb{M}(\alpha-1)} \int_{0}^{T} f(\zeta, \omega(\zeta), \omega(\nu \zeta)) d \zeta \\
& -\frac{t(\alpha-1)}{T \mathbb{M}(\alpha-1) \Gamma(\alpha)} \int_{0}^{T} f(\zeta, \omega(\zeta), \omega(\nu \zeta))(T-\zeta)^{\alpha-1} d \zeta \\
& +\frac{2-\alpha}{\mathbb{M}(\alpha-1)} \int_{0}^{t} f(\zeta, \omega(\zeta), \omega(\nu \zeta)) d \zeta \\
& +\frac{\alpha-1}{\mathbb{M}(\alpha-1) \Gamma(\alpha)} \int_{0}^{t} f(\zeta, \omega(\zeta), \omega(\nu \zeta))(t-\zeta)^{\alpha-1} d \zeta .
\end{aligned}
$$

For the existence and uniqueness of our propose problem (1), we consider the following assumptions to hold:

$\left(B_{1}\right)$ There exists a constant $K_{f}>0$ such that, for any $u, w, \bar{u}, \bar{w} \in J$, one has

$$
|f(t, u, w)-f(t, \bar{u}, \bar{w})| \leq K_{f}\{|u-\bar{u}|+|w-\bar{w}|\} .
$$

$\left(B_{2}\right)$ There exist constants $l_{f}, m_{f}, n_{f}>0$ such that

$$
|f(t, w(t), w(v t))| \leq l_{f}+m_{f}|w(t)|+n_{f}|w(v t)|
$$

Theorem 2 In view of assumption $\left(B_{1}\right)$, the BVP (1) has a unique solution if

$$
\frac{4 K_{f}\left(T \Gamma(\alpha+1)+T^{\alpha}\right)}{\mathbb{M}(\alpha-1) \Gamma(\alpha+1)}<1
$$

Proof Let the operator $\mathbf{F}: X \rightarrow X$ be defined as

$$
\begin{aligned}
\mathbf{F} \omega(t)= & \frac{t w_{1}+w_{0}(T-t)}{T}-\frac{t(2-\alpha)}{T \mathbb{M}(\alpha-1)} \int_{0}^{T} f(\zeta, \omega(\zeta), \omega(\nu \zeta)) d \zeta \\
& -\frac{t(\alpha-1)}{T \mathbb{M}(\alpha-1) \Gamma(\alpha)} \int_{0}^{T} f(\zeta, \omega(\zeta), \omega(\nu \zeta))(T-\zeta)^{\alpha-1} d \zeta
\end{aligned}
$$




$$
\begin{aligned}
& +\frac{2-\alpha}{\mathbb{M}(\alpha-1)} \int_{0}^{t} f(\zeta, \omega(\zeta), \omega(\nu \zeta)) d \zeta \\
& +\frac{\alpha-1}{\mathbb{M}(\alpha-1) \Gamma(\alpha)} \int_{0}^{t} f(\zeta, \omega(\zeta), \omega(\nu \zeta))(t-\zeta)^{\alpha-1} d \zeta
\end{aligned}
$$

To show that $\mathbf{F}$ is a condensing operator, letting $\omega, \bar{\omega} \in X$, one has

$$
\begin{aligned}
\|\mathbf{F} \omega(t)-\mathbf{F} \bar{\omega}(t)\| & \\
= & \max _{t \in J}|\mathbf{F} \omega(t)-\mathbf{F} \bar{\omega}(t)| \\
= & \max _{t \in J} \mid \frac{t(2-\alpha)}{T \mathbb{M}(\alpha-1)} \int_{0}^{T}[f(\zeta, \omega(\zeta), \omega(v \zeta))-f(\zeta, \bar{\omega}(\zeta), \bar{\omega}(v \zeta))] d \zeta \\
& -\frac{t(\alpha-1)}{T \mathbb{M}(\alpha-1) \Gamma(\alpha)} \int_{0}^{T}[f(\zeta, \omega(\zeta), \omega(v \zeta))-f(\zeta, \bar{\omega}(\zeta), \bar{\omega}(v \zeta))](T-\zeta)^{\alpha-1} d \zeta \\
& +\frac{2-\alpha}{\mathbb{M}(\alpha-1)} \int_{0}^{t}[f(\zeta, \omega(\zeta), \omega(\nu \zeta))-f(\zeta, \bar{\omega}(\zeta), \bar{\omega}(v \zeta))] d \zeta \\
& +\frac{\alpha-1}{\mathbb{M}(\alpha-1) \Gamma(\alpha)} \int_{0}^{t}[f(\zeta, \omega(\zeta), \omega(\nu \zeta))-f(\zeta, \bar{\omega}(\zeta), \bar{\omega}(\nu \zeta))](t-\zeta)^{\alpha-1} d \zeta \mid \\
\leq & \frac{4 K_{f}\left(T \Gamma(\alpha+1)+T^{\alpha}\right)}{\mathbb{M}(\alpha-1) \Gamma(\alpha+1)}\|\omega-\bar{\omega}\| .
\end{aligned}
$$

This shows that $\mathbf{F}$ is a condensing map, so it has a unique fixed point. Consequently, our considered problem (1) has a unique solution.

Now consider the operators defined as

$$
\begin{aligned}
\mathbf{G} \omega(t)= & \frac{t w_{1}+w_{0}(T-t)}{T}-\frac{t(2-\alpha)}{T \mathbb{M}(\alpha-1)} \int_{0}^{T} f(\zeta, \omega(\zeta), \omega(\nu \zeta)) d \zeta \\
& -\frac{t(\alpha-1)}{T \mathbb{M}(\alpha-1) \Gamma(\alpha)} \int_{0}^{T} f(\zeta, \omega(\zeta), \omega(\nu \zeta))(T-\zeta)^{\alpha-1} d \zeta \\
\mathbf{H} \omega(t)= & \frac{2-\alpha}{\mathbb{M}(\alpha-1)} \int_{0}^{t} f(\zeta, \omega(\zeta), \omega(\nu \zeta)) d \zeta \\
& +\frac{\alpha-1}{\mathbb{M}(\alpha-1) \Gamma(\alpha)} \int_{0}^{t} f(\zeta, \omega(\zeta), \omega(\nu \zeta))(t-\zeta)^{\alpha-1} d \zeta \\
\mathbf{F} \omega(t)= & \mathbf{G} \omega(t)+\mathbf{H} \omega(t)
\end{aligned}
$$

Theorem 3 If the assumptions $\left(B_{1}\right),\left(B_{2}\right)$ and $0<\frac{2 K_{f}\left(T \Gamma(\alpha+1)+T^{\alpha}\right)}{\mathbb{M}(\alpha-1) \Gamma \alpha+1}<1$ hold, then the proposed problem (1) has at least one solution.

Proof Consider the set $V=\{\omega \in X:\|\omega\| \leq \mathrm{b}\}$. The continuity of $f$ implies that $\mathbf{G}$ is continuous. Now for any $\omega, \bar{\omega} \in V$, one has

$$
\begin{aligned}
\|\mathbf{G} \omega-\mathbf{G} \bar{\omega}\| & =\max _{t \in J}|\mathbf{G} \omega(t)-\mathbf{G} \bar{\omega}(t)| \\
& =\mid-\frac{t(2-\alpha)}{T \mathbb{M}(\alpha-1)} \int_{0}^{T}[f(\zeta, \omega(\zeta), \omega(\nu \zeta))-f(\zeta, \bar{\omega}(\zeta), \bar{\omega}(\nu \zeta))] d \zeta
\end{aligned}
$$




$$
\begin{aligned}
& -\frac{t(\alpha-1)}{T \mathbb{M}(\alpha-1) \Gamma(\alpha)} \\
& \times \int_{0}^{T}[f(\zeta, \omega(\zeta), \omega(\nu \zeta))-f(\zeta, \bar{\omega}(\zeta), \bar{\omega}(\nu \zeta))](T-\zeta)^{\alpha-1} d \zeta \mid \\
\leq & \frac{2 K_{f}\left(T \Gamma(\alpha+1)+T^{\alpha}\right)}{\mathbb{M}(\alpha-1) \Gamma \alpha+1}\|\omega-\bar{\omega}\| .
\end{aligned}
$$

This shows that $\mathbf{G}$ is a contraction. Now for the continuity and compactness of $\mathbf{H}$, considering any $w \in V$, one has

$$
\begin{aligned}
\|\mathbf{H} \omega\|= & \max _{t \in J}|\mathbf{H} \omega(t)| \\
= & \max _{t \in J} \mid \frac{2-\alpha}{\mathbb{M}(\alpha-1)} \int_{0}^{t} f(\zeta, \omega(\zeta), \omega(v \zeta)) d \zeta \\
& +\frac{\alpha-1}{\mathbb{M}(\alpha-1) \Gamma(\alpha)} \int_{0}^{t} f(\zeta, \omega(\zeta), \omega(v \zeta))(t-\zeta)^{\alpha-1} d \zeta \mid \\
\leq & \frac{T \Gamma(\alpha+1)+T^{\alpha}}{\mathbb{M}(\alpha-1) \Gamma(\alpha+1)}\left[l_{f}+\left(m_{f}+n_{f}\right) \mathrm{b}\right] .
\end{aligned}
$$

Hence $G$ is bounded. For continuity letting $t_{1}<t_{2} \in J$, we have

$$
\begin{aligned}
\left|\mathbf{H} \omega\left(t_{2}\right)-\mathbf{H} \omega\left(t_{1}\right)\right| & \\
= & \mid \frac{2-\alpha}{\mathbb{M}(\alpha-1)}\left[\int_{0}^{t_{2}} f(\zeta, \omega(\zeta), \omega(\nu \zeta)) d \zeta-\int_{0}^{t_{1}} f(\zeta, \omega(\zeta), \omega(\nu \zeta)) d \zeta\right] \\
& +\frac{\alpha-1}{\mathbb{M}(\alpha-1) \Gamma(\alpha)} \\
& \times\left[\int_{0}^{t_{2}} f(\zeta, \omega(\zeta), \omega(\nu \zeta))\left(t_{2}-\zeta\right)^{\alpha-1} d \zeta-\int_{0}^{t_{1}} f(\zeta, \omega(\zeta), \omega(\nu \zeta))\left(t_{1}-\zeta\right)^{\alpha-1} d \zeta\right] \mid \\
\leq & \frac{l_{f}+\left(m_{f}+n_{f}\right) \mathrm{b}}{\mathbb{M}(\alpha-1)}\left(t_{2}-t_{1}\right)+\frac{l_{f}+\left(m_{f}+n_{f}\right) \mathrm{b}}{\mathbb{M}(\alpha-1) \Gamma(\alpha+1)}\left(t_{2}^{\alpha}-t_{1}^{\alpha}\right) .
\end{aligned}
$$

This implies that $\left|\mathbf{H} \omega\left(t_{2}\right)-\mathbf{H} \omega\left(t_{1}\right)\right| \rightarrow 0$ as $t_{2} \rightarrow t_{1}$. Also $\mathbf{H}$ is continuous and bounded. Therefore we have $\left\|\mathbf{H} \omega\left(t_{2}\right)-\mathbf{H} \omega\left(t_{1}\right)\right\| \rightarrow 0$ as $t_{2} \rightarrow t_{1}$. Thus $\mathbf{H}$ is completely continuous by the Arzelá-Ascoli theorem. Thus all the requirements of Theorem 1 are obtained so our proposed problem (1) has at least one solution in $V$.

\section{Stability analysis}

This section is concerned with the Ulam type stability for our proposed problem. To achieve the goal, we give some definitions and notions.

Definition 4 The solution of our considered problem (1) is HU stable if there exists a positive number $C_{f}$, such that, for each $\varepsilon>0$ and for each $\omega \in X$ of the inequality

$$
\left|{ }_{0}^{A B C} \mathrm{D}_{t}^{\alpha} \omega(t)-f(t, \omega(t), \omega(v t))\right| \leq \varepsilon, \quad t \in[0, T],
$$

one has a unique solution $\omega^{*} \in X$ of the considered BVP (1) such that

$$
\left\|\omega-\omega^{*}\right\| \leq C_{f} \varepsilon .
$$


It will be generalized Hyers-Ulam (GHU) stable, if we can find

$$
\Phi:(0, \infty) \rightarrow(0, \infty), \quad \Phi(0)=0
$$

such that

$$
\left\|\omega-\omega^{*}\right\| \leq C_{f} \Phi(\varepsilon) .
$$

Remark 1 Let $\omega \in X$ be the solution of inequality given in (7) if and only if we have a function $\beta \in C[0, T]$ which depends on $\omega$ and for each $0 \leq t \leq T$

(i) $|\beta(t)| \leq \varepsilon$;

(ii) ${ }_{0}^{A B C} \mathrm{D}_{t}^{\alpha} \omega(t)=f(t, \omega(t), \omega(v t))+\beta(t)$.

Definition 5 The solution $\omega \in X$ of our proposed problem (1) is Hyers-Ulam-Rassias (HUR) stable with respect to $\psi \in X$ if we can find a real constant $C_{f}>0$ with the property that, for every $\varepsilon>0$ and for each $\omega \in X$ of the inequality

$$
\left|{ }_{0}^{A B C} \mathrm{D}_{t}^{\alpha} \omega(t)-f(t, \omega(t), \omega(\nu t))\right| \leq \psi(t) \varepsilon, \quad \forall t \in[0, T],
$$

one has a unique solution $\omega^{*} \in X$ of the considered BVP (1) such that

$$
\left\|\omega-\omega^{*}\right\| \leq C_{f} \psi(t) \varepsilon
$$

It will be generalized Hyers-Ulam-Rassias (GHUR) stable, if

$$
\left\|\omega-\omega^{*}\right\| \leq C_{f} \psi(t) .
$$

Remark $2 \omega \in X$ is said to be the solution of the inequality given in (8) if and only if we have a function $\beta \in C[0, T]$ which is depending on $\omega$ and for each $0 \leq t \leq T$

(i) $|\beta(t)| \leq \varepsilon \psi(t)$;

(ii) ${ }_{0}^{A B C} \mathrm{D}_{t}^{\alpha} \omega(t)=f(t, \omega(t), \omega(v t))+\beta(t)$.

Lemma 4 Under the Remark 1, the function $\omega \in X$ corresponding to the given problem

$$
\left\{\begin{array}{l}
{ }_{0}^{A B C} \mathrm{D}_{t}^{\alpha} \omega(t)=f(t, \omega(t), \omega(v t))+\beta(t), \quad 1<\alpha \leq 2,0<v<1, \\
\omega(0)=\omega_{0}, \quad \omega(T)=\omega_{1} .
\end{array}\right.
$$

satisfies the relation given by

$$
|\omega(t)-\mathbf{F}(t, \omega(t), \omega(v t))| \leq \mathbf{C}_{\alpha, T} \varepsilon, \quad \text { for all } t \in[0, T],
$$

where

$$
\begin{aligned}
\mathbf{F}(t, \omega(t), \omega(v t))= & \frac{t w_{1}+w_{0}(T-t)}{T}-\frac{t(2-\alpha)}{T \mathbb{M}(\alpha-1)} \int_{0}^{T} f(\zeta, \omega(\zeta), \omega(\nu \zeta)) d \zeta \\
& -\frac{t(\alpha-1)}{T \mathbb{M}(\alpha-1) \Gamma(\alpha)} \int_{0}^{T} f(\zeta, \omega(\zeta), \omega(\nu \zeta))(T-\zeta)^{\alpha-1} d \zeta
\end{aligned}
$$




$$
\begin{aligned}
& +\frac{2-\alpha}{\mathbb{M}(\alpha-1)} \int_{0}^{t} f(\zeta, \omega(\zeta), \omega(\nu \zeta)) d \zeta \\
& +\frac{\alpha-1}{\mathbb{M}(\alpha-1) \Gamma(\alpha)} \int_{0}^{t} f(\zeta, \omega(\zeta), \omega(\nu \zeta))(t-\zeta)^{\alpha-1} d \zeta
\end{aligned}
$$

and

$$
\mathbf{C}_{\alpha, T}=\frac{2\left(T \Gamma(\alpha+1)+T^{\alpha}\right)}{\mathbb{M}(\alpha-1) \Gamma(\alpha+1)}
$$

Proof With the help of Lemma 3, the corresponding problem (9) becomes

$$
\begin{aligned}
\omega(t)= & \frac{t w_{1}+w_{0}(T-t)}{T}-\frac{t(2-\alpha)}{T \mathbb{M}(\alpha-1)} \int_{0}^{T} f(\zeta, \omega(\zeta), \omega(\nu \zeta)) d \zeta \\
& -\frac{t(\alpha-1)}{T \mathbb{M}(\alpha-1) \Gamma(\alpha)} \int_{0}^{T} f(\zeta, \omega(\zeta), \omega(\nu \zeta))(T-\zeta)^{\alpha-1} d \zeta \\
& +\frac{2-\alpha}{\mathbb{M}(\alpha-1)} \int_{0}^{t} f(\zeta, \omega(\zeta), \omega(\nu \zeta)) d \zeta \\
& +\frac{\alpha-1}{\mathbb{M}(\alpha-1) \Gamma(\alpha)} \int_{0}^{t} f(\zeta, \omega(\zeta), \omega(\nu \zeta))(t-\zeta)^{\alpha-1} d \zeta-\frac{t(2-\alpha)}{T \mathbb{M}(\alpha-1)} \int_{0}^{T} \beta(\zeta) d \zeta \\
& -\frac{t(\alpha-1)}{T \mathbb{M}(\alpha-1) \Gamma(\alpha)} \int_{0}^{t} \beta(\zeta)(T-\zeta)^{\alpha-1} d \zeta+\frac{2-\alpha}{\mathbb{M}(\alpha-1)} \int_{0}^{t} \beta(\zeta) d \zeta \\
& +\frac{\alpha-1}{\mathbb{M}(\alpha-1) \Gamma(\alpha)} \int_{0}^{t} \beta(\zeta)(t-\zeta)^{\alpha-1} d \zeta .
\end{aligned}
$$

This implies

$$
|\omega(t)-\mathbf{F}(t, \omega(t), \omega(v t))| \leq \mathbf{C}_{\alpha, T} \varepsilon
$$

Theorem 4 Under the assumption $\left(A_{1}\right)$ along with Lemma 4, the solution of our proposed problem (1) is HU and GHU stable if $1 \neq \mathbf{C}_{\alpha, T}$ holds.

Proof If $\omega$ is any solution and $\omega^{*}$ is a unique solution of problem (1), then one has

$$
\begin{aligned}
\left|\omega(t)-\omega^{*}(t)\right| & =\left|\omega(t)-\mathbf{F}\left(t, \omega^{*}(t), \omega^{*}(v t)\right)\right| \\
& =\left|\omega(t)-\mathbf{F}(t, \omega(t), \omega(v t))+\mathbf{F}(t, \omega(t), \omega(v t))+\mathbf{F}\left(t, \omega^{*}(t), \omega^{*}(v t)\right)\right| \\
& \leq|\omega(t)-\mathbf{F}(t, \omega(t), \omega(v t))|+\left|\mathbf{F}(t, \omega(t), \omega(v t))+\mathbf{F}\left(t, \omega^{*}(t), \omega^{*}(v t)\right)\right| \\
& \leq \mathbf{C}_{\alpha, T} \varepsilon+2 k_{f} \mathbf{C}_{\alpha, T}\left\|\omega-\omega^{*}\right\| .
\end{aligned}
$$

This further implies that

$$
\left\|\omega-\omega^{*}\right\| \leq \frac{\mathbf{C}_{\alpha, T}}{1-2 K_{f} \mathbf{C}_{\alpha, T}} \varepsilon .
$$

Let $C_{f}=\frac{\mathbf{C}_{\alpha, T}}{1-2 K_{f} \mathbf{C}_{\alpha, T}}$, then the solution of the proposed problem (1) is HU stable. Furthermore, if $\Phi(\varepsilon)=\varepsilon$, then the solution is GHU stable. 
Lemma 5 For the BVP (9), the following inequality holds:

$$
|\omega(t)-\mathbf{F}(t, \omega(t), \omega(v t))| \leq \mathbf{C}_{\alpha, T} \varepsilon \Psi(t), \quad \text { for all } t \in[0, T]
$$

Proof We omit the proof as it is straightforward and may be derived like Lemma 4 by using Remark 2.

Theorem 5 Under the assumption $\left(A_{1}\right)$ together with Lemma 5 , the solution of the proposed problem (1) is HUR and GHUR stable if the condition $1 \neq \mathbf{C}_{\alpha, T}$ holds.

Proof One can easily derive the proof like the proof of Theorem 4.

\section{Examples}

In this section, we discuss our result with the help of the following examples.

Example 1 Consider the Dirichlet BVP

$$
\left\{\begin{array}{l}
{ }_{0}^{A B C} \mathrm{D}_{t}^{\frac{3}{2}} \omega(t)=\frac{e^{-t}}{10}+\frac{e^{-\sin (t)}}{40(\omega(t)+1)}+\frac{e^{t-\cos (t)}}{40\left[\omega\left(\frac{t}{3}\right)+2\right]}, \quad t \in[0,1], \\
\omega(0)=0, \quad \omega(1)=0,
\end{array}\right.
$$

clearly $T=1$ and $f\left(t, \omega(t), \omega\left(\frac{1}{3} t\right)\right)=\frac{e^{-t}}{10}+\frac{e^{-\sin (t)}}{40(\omega(t)+1)}+\frac{e^{t-\cos (t)}}{40\left[\omega\left(\frac{t}{3}\right)+2\right]}$ is a continuous function $\forall$ $t \in[0,1]$. Furthermore, let $\omega, \bar{\omega} \in C[J, R]$, then one has

$$
\begin{aligned}
\mid f( & \left.t, \omega(t), \omega\left(\frac{1}{3} t\right)\right)-f\left(t, \bar{\omega}(t), \bar{\omega}\left(\frac{1}{3} t\right)\right) \mid \\
= & \mid\left[\frac{e^{-t}}{10}+\frac{e^{-\sin (t)}}{40(\omega(t)+1)}+\frac{e^{t-\cos (t)}}{40\left[\omega\left(\frac{t}{3}\right)+2\right]}\right] \\
& -\left[\frac{e^{-t}}{10}+\frac{e^{-\sin (t)}}{40(\bar{\omega}(t)+1)}+\frac{e^{t-\cos (t)}}{40\left[\bar{\omega}\left(\frac{t}{3}\right)+2\right]}\right] \mid, \\
\leq & \frac{1}{40}\left\{|\omega(t)-\bar{\omega}(t)|+\mid \omega\left(\frac{1}{3} t\right)-\bar{\omega}\left(\frac{1}{3} t\right)\right\} .
\end{aligned}
$$

So we have $K_{f}=\frac{1}{40}$, and $\alpha=\frac{3}{2}$. Furthermore,

$$
\begin{aligned}
\left|f\left(t, \omega(t), \omega\left(\frac{1}{3} t\right)\right)\right| & =\left|\frac{e^{-t}}{10}+\frac{e^{-\sin (t)}}{40(\omega(t)+1)}+\frac{e^{t-\cos (t)}}{40\left[\omega\left(\frac{t}{3}\right)+2\right]}\right| \\
& \leq \frac{1}{10}+\frac{1}{40}|\omega(t)|+\frac{1}{40}\left|\omega\left(\frac{1}{3} t\right)\right| .
\end{aligned}
$$

Here, $l_{f}=\frac{1}{10}, m_{f}=\frac{1}{40}, n_{f}=\frac{1}{40}$ and $T=1$.

Now

$$
\frac{4 K_{f}\left(T \Gamma(\alpha+1)+T^{\alpha}\right)}{\mathbb{M}(\alpha-1) \Gamma(\alpha+1)}=0.1314<1
$$


Therefore, the conditions of Theorem 2 are satisfied. Thus, the problem (11) has a unique solution. Furthermore,

$$
\frac{2 K_{f}\left(T \Gamma(\alpha+1)+T^{\alpha}\right)}{\mathbb{M}(\alpha-1) \Gamma \alpha+1}=0.0657<1
$$

Hence, the conditions of Theorem 3 also hold. Therefore, (11) has at least one solution. Furthermore, proceeding to verify the stability results, we see that $\mathbf{C}_{\alpha, T}=0.1322 \neq 1$, thus the solution of the mentioned problem (11) is HU stable and consequently GHU stable. Analogously, the conditions of HUR and GHUR stability may be easily derived by taking a nondecreasing function $\Psi(t)=t \in[0,1]$.

Example 2 Consider the inhomogeneous BVP

$$
\left\{\begin{array}{l}
{ }_{0}^{A B C} \mathrm{D}_{t}^{\frac{4}{3}} \omega(t)=\frac{t^{2}+e^{t}}{15}+\frac{e^{-\pi t} \cos (t)}{50+\omega(t)}+\frac{e^{\frac{-t}{2}}}{50+\omega\left(\frac{t}{2}\right)}, \quad t \in[0,1], \\
\omega(0)=e^{\pi}, \quad \omega(1)=\cos \left(\omega\left(\frac{\pi}{3}\right)\right),
\end{array}\right.
$$

$f\left(t, \omega(t), \omega\left(\frac{1}{2} t\right)\right)=\frac{t^{2}+e^{t}}{15}+\frac{e^{-\pi t} \cos (t)}{50+\omega(t)}+\frac{e^{\frac{-t}{2}}}{50+\omega\left(\frac{t}{2}\right)}$ is a continuous function for all $t \in[0,1]$. Furthermore, let $\omega, \bar{\omega} \in C[, R]$, then we have

$$
\begin{aligned}
\mid f( & \left.t, \omega(t), \omega\left(\frac{1}{2} t\right)\right)-f\left(t, \bar{\omega}(t), \bar{\omega}\left(\frac{1}{2} t\right)\right) \mid \\
= & \mid\left[\frac{t^{2}+e^{t}}{15}+\frac{e^{-\pi t} \cos (t)}{50+\omega(t)}+\frac{e^{\frac{-t}{2}}}{50+\omega\left(\frac{t}{2}\right)}\right] \\
& -\left[\frac{t^{2}+e^{t}}{15}+\frac{e^{-\pi t} \cos (t)}{50+\bar{\omega}(t)}+\frac{e^{-\frac{t}{2}}}{50+\bar{\omega}\left(\frac{t}{2}\right)}\right] \mid \\
\leq & \frac{1}{50}\left[|\omega(t)-\bar{\omega}(t)|+\left|\omega\left(\frac{1}{2} t\right)-\bar{\omega}\left(\frac{1}{2} t\right)\right|\right] .
\end{aligned}
$$

Thus from the above, one has $L_{f}=\frac{1}{50}$ and $\alpha=\frac{4}{3}$. Moreover, we have

$$
\begin{aligned}
\left|f\left(t, \omega(t), \omega\left(\frac{1}{2} t\right)\right)\right| & =\left|\frac{t^{2}+e^{t}}{15}+\frac{e^{-\pi t} \cos (t)}{50+\omega(t)}+\frac{e^{\frac{-t}{2}}}{50+\omega\left(\frac{t}{2}\right)}\right| \\
& \leq \frac{1}{15}+\frac{1}{50}|\omega(t)|+\frac{1}{50}\left|\omega\left(\frac{1}{2} t\right)\right|,
\end{aligned}
$$

where $l_{f}=\frac{1}{15}, m_{f}=n_{f}=\frac{1}{50}$ and $T=1$. We obtain

$$
\frac{4 K_{f}\left(T \Gamma(\alpha+1)+T^{\alpha}\right)}{\mathbb{M}(\alpha-1) \Gamma(\alpha+1)}=\frac{\left.4 \Gamma\left(\frac{1}{3}\right)+9\right)}{240 \Gamma\left(\frac{1}{3}\right)}<1 .
$$

Therefore, the conditions of Theorem 2 are satisfied. Thus, the problem (12) has a unique solution. Furthermore,

$$
\frac{2 K_{f}\left(T \Gamma(\alpha+1)+T^{\alpha}\right)}{\mathbb{M}(\alpha-1) \Gamma \alpha+1}=\frac{\left.4 \Gamma\left(\frac{1}{3}\right)+9\right)}{480 \Gamma\left(\frac{1}{3}\right)}<1 .
$$


Hence, the conditions of Theorem 3 also hold. Therefore, (12) has at least one solution. Furthermore, we observed that $\mathbf{C}_{\alpha, T} \neq 1$, hence the solution of the mentioned problem (12) is $\mathrm{HU}$ stable and consequently GHU stable. Along the same line, taking a nondecreasing function $\Psi(t)=1+t$, the condition of HUR and GHUR stability can be derived for the solution of (12).

\section{Conclusion}

We have successfully attained several essential conditions for the existence and stability theory for a class of BVPs involving $A B C$ fractional derivative. By classical fixed point theory like Banach contraction and Krasnoselskii's fixed point theorems, the required results have been established. Furthermore, on using nonlinear analysis some adequate results for different kinds of HU stability have been developed. Providing pertinent examples, the results have been justified.

\section{Acknowledgements}

All authors are very thankful to the reviewers for their useful suggestions. The second author would like to thank Prince Sultan University for funding this research work.

\section{Funding}

Prince Sultan University will provide funding to support this work through research group Nonlinear Analysis Methods in Applied Mathematics (NAMAM) group number RG-DES-2017-01-17.

\section{Availability of data and materials}

Data sharing is not applicable to this article as no data sets were generated or analyzed during the current study.

\section{Competing interests}

The authors declare that they have no competing interests.

\section{Authors' contributions}

All authos equally contributed to this manuscript and approved the final version.

\section{Author details}

'Department of Mathematics, University of Malakand, 18000 Dir(L), Khyber Pakhtunkhwa, Pakistan. ${ }^{2}$ Department of Mathematics and General Sciences, Prince Sultan University, Riyadh, Saudi Arabia. ${ }^{3}$ Department of Medical Research, China Medical University, Taichung 40402, Taiwan. ${ }^{4}$ Department of Computer Science and Information Engineering, Asia University, Taichung, Taiwan. ${ }^{5}$ Department of Mathematics, Shaheed Benazir Bhutto University, Sheringal, 18000 Dir(U),

Pakistan. ${ }^{6}$ Department of Mathematics \& Statistics, University of Swat, 18000 Swat, Pakistan.

\section{Publisher's Note}

Springer Nature remains neutral with regard to jurisdictional claims in published maps and institutional affiliations.

Received: 14 May 2020 Accepted: 27 July 2020 Published online: 05 August 2020

\section{References}

1. Ishteva, M.K.: Properties and application of the Caputo Fractional operator. Dept. Math. Univ. Karlsruhe (2005)

2. Zhou, Y.: Basic Theory of Fractional Differential Equations. World Scientific, Singapore (1964)

3. Miller, K.S., Ross, B.: An Introduction to the Fractional Calculus and Fractional Differential Equations, Wiley-Interscience Publications. Wiley, New York (1993)

4. Podlubny, I.: Fractional Differential Equations, Mathematics in Science and Engineering. Academic Press, New York (1999)

5. Caputo, M.: Linear models of dissipation whose $Q$ is almost frequency independent. Geophys. J. Int. 13, 529-539 (1967)

6. Hilfer, R.: Applications of Fractional Calculus in Physics. World Scientific, Singapore (2000)

7. Kilbas, A.A., Marichev, O.I., Samko, S.G.: Fractional Integrals and Derivatives. Gordon \& Breach, Yverdon (1993)

8. Kilbas, A.A., Srivastava, H.M., Trujillo, J.J.:Theory and Applications of Fractional Differential Equations. North-Holland Mathematics Studies, vol. 204. Elsevier, Amsterdam (2006)

9. Lakshmikantham, V., Leela, S., Vasundhara, J.: Theory of Fractional Dynamic Systems. Cambridge Academic Publishers, Cambridge (2009)

10. Wu, R.C., Hei, X.D., Chen, L.P.: Finite-time stability of fractional-order neural networks with delay. Commun. Theor. Phys. 60, 189-193 (2013)

11. Buhmann, M., Iserles, A.: Stability of the discretized pantograph differential equation. Math. Comput. 60(202), 575-589 (1993) 
12. Cao, J., Lu, J.: Adaptive synchronization of neural networks with or without time-varying delay. Chaos 16(1), 013133 (2006)

13. Magin, R.L.: Fractional calculus models of complex dynamics in biological tissues. Comput. Math. Appl. 59, 1586-1593 (2010)

14. Tunç, C., Golmankhaneh, A.K., Branch, U.: On stability of a class of second alpha-order fractal differential equations. AIMS Math. 5(3), 2126-2142 (2020)

15. Khan, R.A., Shah, K.: Existence and uniqueness of solutions to fractional order multi-point boundary value problems. Commun. Appl. Anal. 19, 515-526 (2015)

16. Atangana, A., Baleanu, D.: New fractional derivatives with nonlocal and non-singular kernel. Theor. Appl. Heat Transf. Model, Therm. Sci. 20, 763-769 (2016)

17. Djida, J.D., Atangana, A., Area, I.: Numerical computation of a fractional derivative with non-local and non-singular kernel. Math. Model. Nat. Phenom. 12(3), 4-13 (2017)

18. Algahtani, O.: Comparing the Atangana-Baleanu and Caputo-Fabrizio derivative with fractional order Allen Cahn model. Chaos Solitons Fractals 89, 552-559 (2016)

19. Trigeassou, J.C., et al.: A Lyapunov approach to the stability of fractional differential equations. Signal Process. 91(3), 437-445 (2011)

20. Lijun, G., Wang, D., Wang, G.: Further results on exponential stability for impulsive switched nonlinear time-delay systems with delayed impulse effects. Appl. Math. Comput. 268, 186-200 (2015)

21. Stamova, I.: Mittag-Leffler stability of impulsive differential equations of fractional order. Q. Appl. Math. 73(3), 525-535 (2015)

22. Ulam, S.M.: Problems in Modern Mathematics. Wiley, New York (1940)

23. Tunç, C., Biçer, E.: Hyers-Ulam-Rassias stability for a first order functional differential equation. J. Math. Fund. Sci. 47(2), 143-153 (2015)

24. Ulam, S.M.: Problems in Modern Mathematics. Wiley, New York (1940)

25. Ulam, S.M.: A Collection of Mathematical Problems. Interscience, New York (1960)

26. Hristova, S., Tunç, C.: Stability of nonlinear Volterra integro-differential equations with Caputo fractional derivative and bounded delays. Electron. J. Differ. Equ. 30(1), 1 (2019)

27. Ali, A., Shah, K., Abdeljawad, T.: Study of implicit delay fractional differential equations under anti-periodic boundary conditions. Adv. Differ. Equ. 2020(1), 1 (2020)

28. Coronel-Escamilla, A., Gómez-Aguilar, J.F.: A novel predictor-corrector scheme for solving variable-order fractional delay differential equations involving operators with Mittag-Leffler kernel. Discrete Contin. Dyn. Syst., Ser. S 13(3), 561-574 (2020)

29. Balachandran, K., Kiruthika, S., Trujillo, J.J.: Existence of solution of nonlinear fractional pantograph equations. Acta Math. Sci. 33(3), 712-720 (2013)

30. Tohidi, E., Bhrawy, A.H., Erfani, K.: A collocation method based on Bernoulli operational matrix for numerical solution of generalized pantograph equation. Appl. Math. Model. 37(6), 4283-4294 (2013)

31. Abdeljawad, T.: A Lyapunov type inequality for fractional operators with nonsingular Mittag-Leffler kernel. J. Inequal. Appl. 2017, 130 (2017)

32. Burton, T.A.: A fixed point theorem of Krasnoselskii. Appl. Math. Lett. 11, $85-88$ (1998)

\section{Submit your manuscript to a SpringerOpen ${ }^{\circ}$ journal and benefit from:}

- Convenient online submission

- Rigorous peer review

- Open access: articles freely available online

- High visibility within the field

Retaining the copyright to your article

Submit your next manuscript at $>$ springeropen.com 